\title{
Cardiac arrest in the prone position caused by central venous cannulation-induced cardiac tamponade
}

\author{
Nitasha Mishra ${ }^{1}$, Shalendra Singh ${ }^{2}$, Anirudh Elayat $^{1}$, and Ashutosh Kaushal ${ }^{3}$ \\ ${ }^{1}$ All India Institute of Medical Sciences, Bhubaneshwar, Orissa, ${ }^{2}$ Department of Anesthesiology and Critical Care, \\ Armed Forces Medical College, Pune, ${ }^{3}$ All India Institute of Medical Sciences, Rishikesh, Uttarakhand, India
}

Intraoperative prone positioning causes various physiological cardiovascular and respiratory alterations that can rarely lead to cardiac arrest (CA). Intraoperative CA is usually attributable to preexisting diseases or intraoperative complications in most patients. Almost all patients undergoing a neurosurgical procedure require central venous cannulation (CVC) prior to surgery for central venous pressure monitoring, fluid/blood product administration, and administration of various drugs, including ionotropes. Cardiac tamponade (CaT) is an uncommon but potentially life-threatening complication, with an incidence of $0.0001 \%$ to $1.4 \%$ but a mortality rate of $40 \%-100 \%$ in the supine position $[1,2]$. However, CVC-induced CaT resulting in CA in the prone position has not been reported. Misdiagnosis at this point can lead to difficulty in patient revival. We report a case of successful resuscitation of CVC-induced CaT in which the patient had sudden and rapid hemodynamic deterioration soon after being turned to the prone position after the administration of general anesthesia (GA).

Patient consent was obtained for this report. A 35-year-old woman, weighing $65 \mathrm{~kg}$, who had a C2-C3 intradural extramedullary meningioma with no known comorbidities or history

Corresponding author: Shalendra Singh, D.M.

Department of Anesthesiology and Critical Care, Armed Forces Medical College, Pune 411040, India

Tel: +91-9466332207, Fax: +91-020-26306036

Email: drsinghafmc@gmail.com

ORCID: https://orcid.org/0000-0002-1112-3431

Received: March 16, 2019.

Revised: March 24, 2019 (1st); March 28, 2019 (2nd).

Accepted: April 3, 2019.

Korean J Anesthesiol 2019 August 72(4): 394-395

https://doi.org/10.4097/kja.19105 of previous surgeries was indicated for laminectomy and tumor excision. She was given GA in accordance with the institutional protocol. Postinduction, her vitals were stable, with a heart rate (HR) of $85 / \mathrm{min}$ and invasive blood pressure (IBP) of 106/50 $\mathrm{mmHg}$. The right subclavian vein was cannulated using a $7-\mathrm{Fr}$ triple-lumen catheter and secured with sutures after confirming backflow through all the ports. Fifteen minutes after proning the patient over Wilson's frame and stabilizing the head using Mayfield pins, a decrease in end-tidal $\mathrm{CO}_{2}\left(\mathrm{ETCO}_{2}\right)$ from $32 \mathrm{mmHg}$ to $13 \mathrm{mmHg}$, along with a sudden decrease in IBP from 110/58 $\mathrm{mmHg}$ to $40 / 22 \mathrm{mmHg}$, was observed for 2-3 min, which eventually led to CA. Cardiopulmonary resuscitation (CPR) was immediately started in the prone position, and intravenous adrenaline of $1 \mathrm{mg}$ was administered. The rapidity of the event led us to assume venous air embolism (due to surgical pins) or tension pneumothorax (due to CVC) to be the most probable causes of the unusual event. After 1 minute of CPR in the prone position, the patient was turned to the supine position and CPR was continued. Rapid fluid boluses were given, and noradrenaline infusion of $0.5 \mu \mathrm{g} / \mathrm{kg} / \mathrm{min}$ was started. An attempt was made to aspirate air through the central venous catheter, which showed no aspirate. Within 1-2 min of turning the patient to the supine position and continuing $\mathrm{CPR}$, she was revived. Monitoring revealed a HR of $130 / \mathrm{min}$, IBP of $90 / 50 \mathrm{mmHg}$ on noradrenaline infusion of $0.5 \mu \mathrm{g} / \mathrm{kg} / \mathrm{min}$, and electrocardiography revealed sinus tachycardia. The chest radiography and transthoracic echocardiography performed in the operating theater revealed a pericardial collection that caused obstructive shock with a severely restricted ventricular end-diastolic volume (Fig. 1A). A provisional diagnosis of CaT was made, and the patient was transferred to the surgical intensive care unit, where the cardiologist performed bedside echocardiographic pericardiocentesis and aspirated approximately $50 \mathrm{ml}$ of sero sanguineous fluid (Fig.

(c) This is an open-access article distributed under the terms of the Creative Commons Attribution Non-Commercial License (http://creativecommons.org/ licenses/by-nc/4.0/), which permits unrestricted non-commercial use, distribution, and reproduction in any medium, provided the original work is properly cited. 

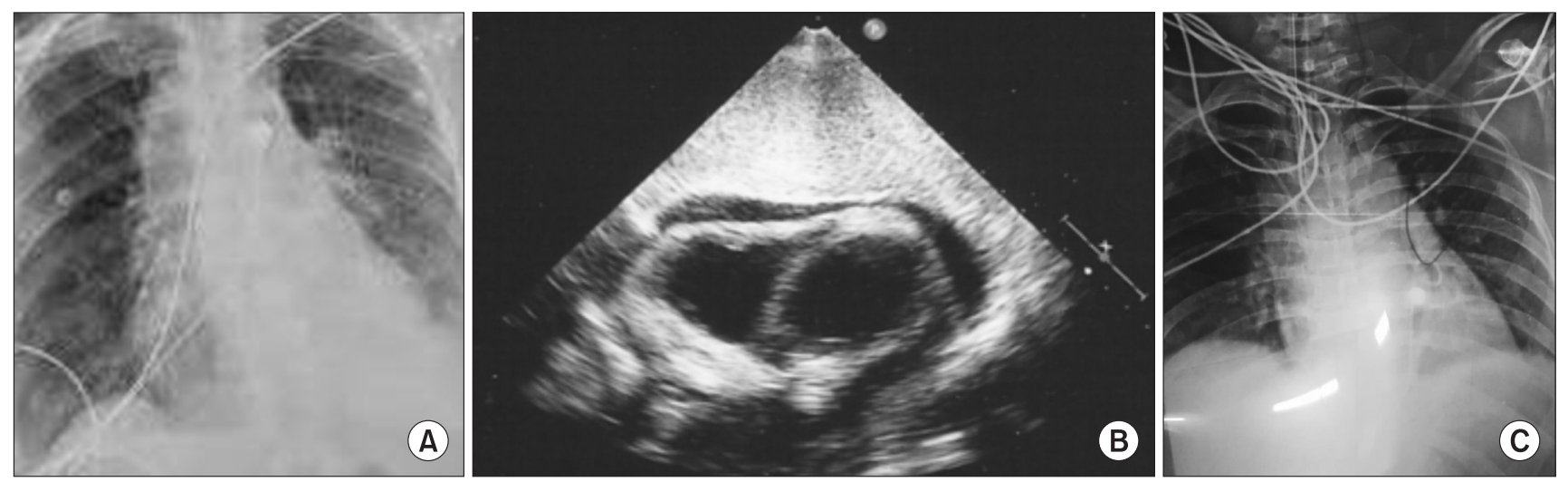

Fig. 1. (A) Chest radiograph showing pericardial ollection in the operation theater. (B) Echocardiographic imageshowing pericardial collection during pericardiocentesis. (C) Chest radiograph showing pericardial collectionwith a pigtail catheter in situ.

1B). The vitals showed an IBP of $180 / 70 \mathrm{mmHg}$; thus, vasopressor infusion was discontinued. The patient was then transferred to the catheterization laboratory, where fluoroscopy-guided pigtail catheterization of the pericardium with further aspiration of $150 \mathrm{ml}$ of hemorrhagic fluid was performed (Fig. 1C). The tear in the right atrial chamber was located using a radio-opaque dye through the distal port of the central venous catheter, and a defect of $0.5 \mathrm{~mm}$ with evidence of leakage into the pericardial space was identified. The pigtail catheter was left in place for 48 $\mathrm{h}$ with constant negative suction to drain out another $180 \mathrm{ml}$ of fluid over $48 \mathrm{~h}$, after which the catheter was removed. The patient was extubated on the third day.

Although intraoperative CA is rare in the prone position, anesthesiologists should be aware of CaT, which can be one of the causes. Timely diagnosis requires a high index of suspicion in the setting of prone positioning with unexplained hemodynamic deterioration in a recently placed CVC. The use of point-of-care ultrasonography is indispensable and lifesaving in such cases.

\section{Conflicts of Interest}

No potential conflict of interest relevant to this article was reported.

\section{Author Contributions}

Nitasha Mishra (Investigation)

Shalendra Singh (Conceptualization; Formal analysis)

Anirudh Elayat (Investigation)

Ashutosh Kaushal (Formal analysis)

\section{ORCID}

Nitasha Mishra, https://orcid.org/0000-0002-3558-2755

Shalendra Singh, https://orcid.org/0000-0002-1112-3431

Anirudh Elayat, https://orcid.org/0000-0002-4090-5504

Ashutosh Kaushal, https://orcid.org/0000-0001-8552-7861

\section{References}

1. Datta R, Purohit G, Agrawal J, Singh S. Pericardial tamponade: Rare complication of subclavian vein cannulation. Med J Armed Forces India 2018; 74: 294-6.

2. Booth SA, Norton B, Mulvey DA. Central venous catheterization and fatal cardiac tamponade. Br J Anaesth 2001; 87: 298-302. 